\title{
Saccharomyces cerevisiae, Non-Saccharomyces Yeasts and Lactic Acid Bacteria in Sequential Fermentations: Effect on Phenolics and Sensory Attributes of South African Syrah Wines
}

\author{
P.P. Minnaar ${ }^{1,2, *}$, H.W. du Plessis ${ }^{1,2}$, V. Paulsen ${ }^{4}$, N. Ntushelo ${ }^{3}$, N.P. Jolly ${ }^{1}$, M. du Toit ${ }^{2}$ \\ (1) ARC Infruitec-Nietvoorbij, Private Bag X5026, Stellenbosch 7600, South Africa \\ (2) Institute for Wine Biotechnology \& Department of Viticulture and Oenology, Stellenbosch University, Private Bag X1, \\ Matieland 7602, South Africa \\ (3) ARC Biometry, Private Bag X5026, Stellenbosch 7600, South Africa \\ (4) Process Engineering, Stellenbosch University, Private Bag X1, Matieland 7602, South Africa
}

Submitted for publication: March 2017

Accepted for publication: May 2017

Keywords: Anthocyanins, flavan-3-ols, flavonols, Hanseniaspora uvarum, Lactobacillus plantarum, malolactic fermentation, Metschnikowia pulcherrima, Oenococcus oeni, phenolic acids, Saccharomyces cerevisiae

\begin{abstract}
Wine consumers predominantly use visual, sensory and textual descriptors as quality/preference indicators to describe olfactory sensations. In this study, different wines were analysed to generate relevant chemical and sensory characterisation data and attributes. Sequential inoculation of Syrah grape must was performed with a combination of Saccharomyces yeast, non-Saccharomyces yeasts and lactic acid bacteria for the possible improvement of Syrah wine quality. Selected anthocyanins, flavan-3-ols, flavonols and phenolic acids were quantified in Syrah wines using the reversed-phase high-performance liquid chromatography photodiode array detection (RP-HPLC-DAD) technique. Sensory (descriptive evaluation) and physicochemical/oenological parameters (Winescan ${ }^{\circledR}$ and OenoFoss ${ }^{\mathrm{TM}}$ ) results were compared to phenolic compound concentrations. Phenolic compound concentrations increased in Syrah wines made with a combination of a Saccharomyces reference yeast, non-Saccharomyces yeasts and lactic acid bacteria. Syrah wines made with a combination of Metschnikowia pulcherrima + Saccharomyces cerevisiae + Oenococcus oeni, and M. pulcherrima + S. cerevisiae + Lactobacillus plantarum, had higher flavonol concentrations compared to wines made without lactic acid bacteria. Syrah wines made with a combination of Saccharomyces cerevisiae (Sc) + Oenococcus oeni (LAB1) were highest in phenolic acid concentrations. Syrah wines made with a combination of $M$. pulcherrima + S. cerevisiae + L. plantarum had higher total anthocyanins than wines made without lactic acid bacteria. Syrah wine sensory attributes, viz. mouthfeel and astringency, correlated with a combination of lactic acid bacteria and yeast treatments. Syrah wines made with a combination of yeast and lactic acid bacteria (LAB) scored highest in overall quality. Indications are that the $S$. cerevisiae reference yeast retained more phenolic compounds during fermentation when compared to wines made with a combination of non-Saccharomyces yeasts and LAB. The improved red colour of Syrah wines may be achieved by sequential inoculation with non-Saccharomyces yeast and LAB. This could be beneficial where winemakers use grape cultivars with low anthocyanin levels in the grape skin to produce wines of improved quality.
\end{abstract}

\section{INTRODUCTION}

The South African wine industry is an important agricultural entity due to its contribution to the economy of the country by creating employment opportunities and contributing to local tourism ( $c a$. R36 billion GDP; South African Wine Industry and Information Systems (SAWIS), 2015; WOSA, 2017). On-going improvement in wine quality is imperative for growth in the wine industry. Improvements include microbiological aspects, such as choices for yeast and lactic acid bacteria (LAB) for wine production. Yeasts can improve the sensorial properties of wine via the production of metabolites that affect the colour, aroma and structure of wine (Morata et al., 2012). Wine colour and structure can be affected by changes in the phenolic compound concentration. Grape must is normally inoculated with Saccharomyces cerevisiae yeast for commercial winemaking. Caridi et al. (2004) have shown that $S$. cerevisiae wine yeasts can

\footnotetext{
*Corresponding author: E-mail address: minnaarp@arc.agric.za

Acknowledgements: The authors thank the ARC, Winetech and the National Research Foundation of South Africa (THRIP programme; grant numbers UID 71526 and 86086) for funding. The opinions, findings and conclusions expressed in this publication are those of the authors. The National Research Foundation accepts no liability in this regard. Mmes V. van Breda, P. Adonis and M. Vermeulen, as well as Messrs J. Hoff and G. Harold are thanked for technical assistance.
} 
decrease the phenolic compound concentrations of wine. The use of Saccharomyces and non-Saccharomyces yeasts as mixed starter cultures is of increasing interest for quality enhancement and the improved complexity of wine (Jolly et al., 2014; Padilla et al., 2016). Morata et al. (2012) reported on the use of non-Saccharomyces yeast strains alone or in combination with $S$. cerevisiae in mixed fermentations that showed improved sensorial properties of wine and increased phenolic compound concentrations.

Malolactic fermentation (MLF) is an enzymatic reaction performed by LAB, whereby L-malic acid is decarboxylated to L-lactic acid and $\mathrm{CO}_{2}(\mathrm{Du}$ Toit et al., 2010; Lerm et al., 2010; Du Plessis et al., 2017). During MLF, wine acidity is reduced, the flavour profile is modified, and the microbiological stability of the wine increases. Malolactic fermentation and LAB can also affect grape phenolic compound concentrations (Hernández et al., 2006; 2007). The principal phenolic compounds that are transformed by different LAB include hydroxycinnamic acids and their derivatives, flavonols and their glycosides, flavan-3-ol monomers $[(+)$-catechin and (-)-epicatechin] and flavan-3ol oligomers [procyanidins] (García-Ruiz et al., 2008). The hydroxycinnamic acids (e.g. gallic acid and (+)-catechin) are affected and degraded by certain LAB and $S$. cerevisiae strains (Hernández et al., 2006; García-Ruiz et al., 2008). The effect of MLF on phenolic compound concentrations in red wine is directly linked to the metabolism of LAB (Hernández et al., 2006). Phenolic compounds, in turn, can affect the growth and metabolism of LAB (Alberto et al., 2007) and also the occurrence and progression of MLF (Vivas et al., 1997a). Phenolic acids inhibit the growth of LAB (Reguant et al., 2000; Knoll et al., 2008), while flavan3-ols and flavonols can stimulate the growth of specifically Oenococcus oeni (Vivas et al., 1997a; Rozès et al., 2003).

Grape phenolic compounds play an important role in the visual and gustative quality of red wine (BautistaOrtín et al., 2007). These compounds also contribute to the complexity and stability of the wine (Caridi et al., 2004). Red wine colour intensity plays an important role in the perceived sensory quality and depends on the concentrations of phenolic compounds, viz. anthocyanins (colour), flavan3-ol monomers and oligomers (astringency, mouthfeel and bitterness), flavonols, phenolic acids and other phenolic compounds. These phenolic compounds are extracted from the grape skins into the must and ultimately into the wine during grape skin maceration and grape pomace contact (Mateus et al., 2002; Lorrain et al., 2013). Anthocyanin monomers are responsible for the development of the red colour, whilst their-acetylated and coumaroylated derivatives provide stability to the red colour of young wines (RibéreauGayon et al., 2006; Gawel \& Godden, 2008). Wine colour also depends on the polymerisation (modification) of anthocyanins during vinification, wine storage and wine maturation (Schwarz et al., 2003). Phenolic compound concentrations in wine can also vary according to grape cultivar, viticultural practices, skin maceration temperature (Cheynier et al., 1997; Ribéreau-Gayon et al., 2006) and grape pomace contact time (Mazza \& Francis, 1995;
Ribéreau-Gayon et al., 2006; Song et al., 2015).

The diffusion of grape phenolic compounds from the grape skin to the grape must is based on the molecular structure of the particular phenolic compound, regardless of whether the diffusion kinetics are affected by the formation of acetaldehyde or ethanol (Di Stefano et al., 1994). Yeasts release secondary metabolites, such as pyruvic acid and acetaldehyde, into the fermentation medium, some of which react with anthocyanins (Eglinton et al., 2004; Fulcrand et al., 2006). This reaction can lead to the stabilisation of anthocyanins during the maturation of wine. Yeast lees can modify the colour of wine, either by the formation of weak and reversible yeast-anthocyanin interactions, or by anthocyanin and yeast cell wall mannoprotein interactions (Morata et al., 2003; Morata et al., 2012). However, anthocyanins are removed from the fermentation medium by the yeast during fermentation through yeast cell adsorption (Medina et al., 2005; Burns \& Osborne, 2015). Adsorption of anthocyanins to the yeast cell wall is attributed to the porous peptide/polysaccharide component of the yeast cell wall (Hernández et al., 2003). Adsorption is followed by enzymatic hydrolysis involving yeast periplasmic anthocyanin-ß-D-glucoside activity (ß-glucosidase activity) or the cleavage of the 3-O-glucoside moiety, which results in a decolourising activity or anthocyanin degradation (Manzanares et al., 2000; Hernández et al., 2003). Wine lees, grape residue and yeast cell sediment are therefore pigmented upon completion of alcoholic fermentation. The degree of anthocyanin removal can be an important factor in determining the wine quality. Factors such as fermentation temperature, percentage ethanol and $\mathrm{SO}_{2}$ levels in the wine can also affect the adsorption rate of anthocyanins by yeast cell walls (Salmon, 2006; Nguela et al., 2015). Yeast-anthocyanin interaction can also contribute to the stabilisation of wine colour through the participation of pyranoanthocyanin formation during fermentation (Morata et al., 2003).

The aim of this study was to determine whether the interactions of mixed culture fermentations of $S$. cerevisiae, non-Saccharomyces yeasts and LAB affect the concentrations of anthocyanins, flavonols, flavan-3-ols and phenolic acids in Syrah wines. The effects of these interactions on selected sensory attributes were also investigated.

\section{MATERIALS AND METHODS}

\section{Starter cultures used for fermentation}

The commercial S. cerevisiae yeast VIN 13 (Anchor Wine Yeast, South Africa), one Hanseniaspora uvarum yeast strain (ARC Infruitec-Nietvoorbij culture collection), one Metschnikowia pulcherrima yeast strain (ARC InfruitecNietvoorbij culture collection), and two LAB strains, viz. O. oeni (Viniflora ${ }^{\circledR}$ oenos, Chr. Hansen, Denmark) and Lactobacillus plantarum (Enoferm V22, Lallemand, France), were evaluated in mixed-culture fermentations. The following abbreviations are used: $S$. cerevisiae $(\mathrm{Sc})$, H. uvarum $(\mathrm{Hu})$, M. pulcherrima $(\mathrm{Mp})$, O. oeni $(\mathrm{LAB} 1)$ and L. plantarum (LAB2). 


\section{Fermentation procedure}

Syrah grapes were handpicked from grapevines planted in a northwest-southeast row direction and trained to a VSP trellis on the Nietvoorbij research farm $(33.914865,18.861047)$ near Stellenbosch, South Africa. Grapes were destemmed and crushed. Equal portions of grape skins and juice were divided into $70 \mathrm{~L}$ fermentation bins. Fermentations were carried out in a temperature-controlled room at $c a .24^{\circ} \mathrm{C}$ using a standardised winemaking protocol as described by Minnaar et al. (2015). Treatments included $S$. cerevisiae on its own (reference treatment), $S$. cerevisiae in combination with non-Saccharomyces yeasts, and S. cerevisiae and nonSaccharomyces yeasts in combination with LAB (Table 1). All treatments were performed in triplicate. Metschnikowia pulcherrima and Hanseniaspora uvarum were inoculated on day 0 as wet cultures at a concentration of $8.4 \times 10^{5}$ and $6.4 \times 10^{5}$ cells $/ \mathrm{mL}$, respectively. Saccharomyces cerevisiae yeasts $(0.3 \mathrm{~g} / \mathrm{L}$ active dry yeast $)$ were added 24 hours later (day 1) to complete the alcoholic fermentation, whereas $0.3 \mathrm{~g} / \mathrm{L}$ of the active dry yeast was added on day 0 for the control treatment. The fermentation caps were punched down two times per day and all treatments had the same grape-pomace contact time. After the completion of alcoholic fermentation and separation of the wine from the grape pomace, LAB1 and LAB2 were inoculated according to the suppliers' recommendations. Wines were racked off the lees and the total $\mathrm{SO}_{2}$ adjusted to $85 \mathrm{mg} / \mathrm{L}$ after the completion of MLF. Malolactic fermentation was considered complete when L-malic acid was less than $0.2 \mathrm{~g} / \mathrm{L}$. Wines were stored at $15^{\circ} \mathrm{C}$ until required for analysis. Total soluble solids, total acidity, malic acid, yeast assimilable nitrogen (YAN) and volatile acidity were analysed in the Syrah juice using a Foss ${ }^{\circledR}$ Winescan (IWBT, Stellenbosch University, Stellenbosch). Residual sugar, malic acid, $\mathrm{pH}$, total acidity, ethanol $(\%, v / v)$ and volatile acidity were determined on the finished wine using an Oenofoss ${ }^{\mathrm{TM}}$ analyser (FOSS Analytical A/S, Denmark).

\section{Wine phenolic compounds}

Wine phenolic compounds were quantified using a reversedphase high-performance liquid chromatography-photodiode array detection (RP-HPLC-DAD) technique as described by Waterhouse et al. (1999) and Minnaar et al. (2015). Monomeric anthocyanins, flavan-3-ols, flavonols and phenolic acids were measured at absorbance wavelengths of $520 \mathrm{~nm}, 280 \mathrm{~nm}, 360 \mathrm{~nm}$ and $316 \mathrm{~nm}$ respectively. The separation and quantification of the compounds were performed based on calibration curves using commercially available standards and ultraviolet absorbance spectra. Wine samples were filtered through a $0.22 \mu \mathrm{m}$ nylon membrane syringe filter prior to HPLC analysis.

\section{Sensory analyses}

A panel of 24 judges experienced in wine evaluation evaluated the wines 16 months after bottling. Wines were evaluated for acidity, mouthfeel, astringency, bitterness and overall quality. Sensory evaluations took place in tasting booths and $c a .30 \mathrm{~mL}$ of coded wine was presented in random order to each judge in a standard international winetasting glass. A $10 \mathrm{~cm}$ unstructured line scale was used for

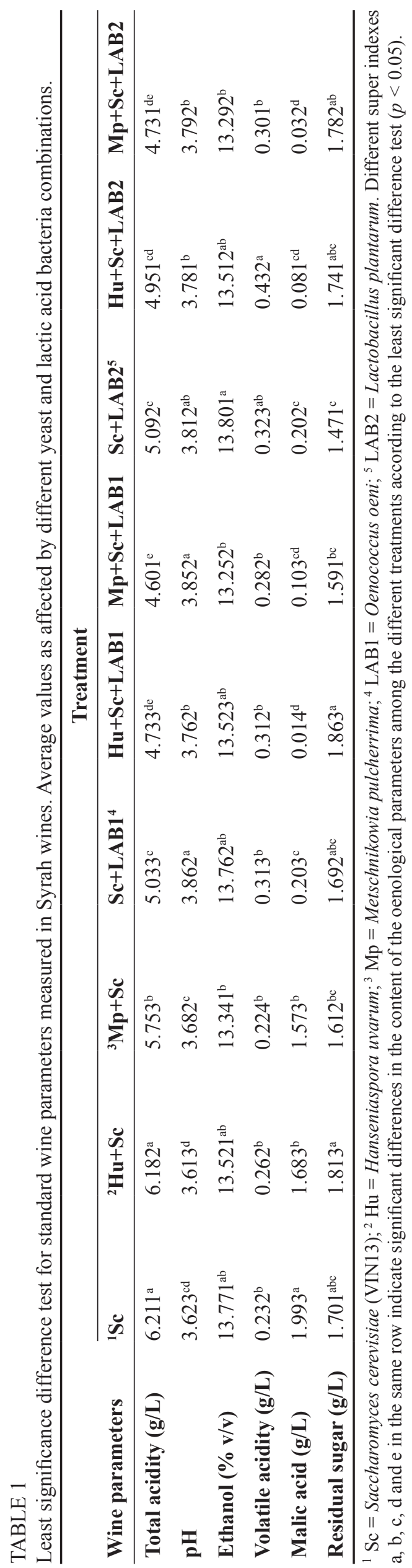


scoring. Judges were asked to rate the attributes from low to high (acidity), thin to full (mouthfeel), and undetectable to prominent (bitterness). Overall quality was rated from unacceptable to excellent.

\section{Statistical analyses}

The resulting data was tested for normality by the method of Shapiro and Wilk (1965). The data was subjected to analysis of variance (ANOVA) using SAS version 9.2 (SAS Institute Inc., n.d.). Student's $t$-least significant difference values (LSD) were calculated at the $5 \%$ probability level to facilitate comparison between treatment means (Ott, 1998). Means within data sets that differed at the $5 \%$ probability level were considered significantly different.

\section{RESULTS}

\section{Treatment effect on wine physicochemical parameters}

Prior to inoculation the Syrah grape juice was analysed for total soluble solids ( $241.00 \mathrm{~g} / \mathrm{L})$, total acidity $(7.43 \mathrm{~g} / \mathrm{L})$, malic acid $(3.10 \mathrm{~g} / \mathrm{L})$, volatile acidity $(0.44 \mathrm{~g} / \mathrm{L})$ and YAN (133.00 mg/L).

There were significant differences among the treatments, i.e. the yeast and bacteria combinations and the yeast only, in terms of wine parameters measured (Table 1). Total acidity was significantly different for wines that underwent MLF compared to those that did not. Th lowest total acidity levels were evident in wines made with a combination of $\mathrm{Mp}+$ Sc + LAB1. Wines that underwent MLF also showed the highest $\mathrm{pH}$ values. There were no significant differences between the reference wines ( $S$. cerevisiae only) and the yeast/LAB combination wines for ethanol levels. Wines made with $S$. cerevisiae only and a combination of $\mathrm{Mp}+\mathrm{Sc}$ had the lowest volatile acidity levels. Wines that underwent MLF showed highest levels of volatile acidity. All wines fermented to dryness, i.e. $<4 \mathrm{~g} / \mathrm{L}$. There were no significant differences between wines that underwent MLF and wines made with yeast only in terms of residual sugar, except for the wines made with a combination of Sc + LAB2, which were lowest in residual sugar.

\section{Treatment effect on phenolic compounds}

There were significant differences among the wine treatments in terms of phenolic compound concentrations (Table 2). Significant differences for $(+)$-catechin were noted among wines made with a combination of yeast only, viz. $\mathrm{Hu}+\mathrm{Sc}$, and a combination of yeast and LAB, viz. $\mathrm{Hu}+\mathrm{Sc}+\mathrm{LAB} 1$, $\mathrm{Sc}+\mathrm{LAB} 2$ and $\mathrm{Mp}+\mathrm{Sc}+\mathrm{LAB} 2$.

Caffeic acid was highest in wines made with a combination of $\mathrm{Mp}+\mathrm{Sc}+\mathrm{LAB} 1$ and lowest in yeast-only wines. Significant differences in caffeic acid were noted among wines made with a combination of Sc + LAB1, Mp + $\mathrm{Sc}+\mathrm{LAB} 1$ and Sc only. Significant differences in gallic acid concentration were not evident among the nine treatments. Gallic acid concentrations were lowest in wines made with the $\mathrm{Mp}+\mathrm{Sc}$ combination. Significant differences were shown for $p$-coumaric acid concentrations among wines made with yeast only and wines that underwent MLF. Reference wines (Sc only) were lowest in $p$-coumaric acid concentrations. Ferulic acid concentrations were not significantly different among the wines. Wines made with a $\mathrm{Hu}+\mathrm{Sc}$ combination showed the lowest concentrations of caffeic acid, and wines made with a $\mathrm{Mp}+\mathrm{Sc}+\mathrm{LAB} 1$ combination proved lowest in ferulic acid concentrations.

There were no significant differences in flavonol concentrations among treatments, except wines made with Sc only, which were lowest in kaempferol concentrations. Kaempferol concentrations were highest in wines that underwent MLF.

Delphinidin and peonidin 3-O-glucoside concentrations were significantly different in wines made with a $\mathrm{Hu}+\mathrm{Sc}$ $+\mathrm{LAB} 2$ and $\mathrm{Mp}+\mathrm{Sc}+\mathrm{LAB} 2$ combinations, compared to wines made with $\mathrm{S}$. cerevisiae only. Petunidin 3-O-glucoside concentrations were not significantly different among the wines. Malvidin 3-O-glucoside concentrations were higher in wines that underwent MLF (except wines made with a combination of $\mathrm{Sc}+\mathrm{LAB} 2)$ compared to wines made with yeast only. Delphinidin 3-O-(6-acetyl) glucosides were lowest in wines that underwent MLF, except wines made with the combination of $\mathrm{Mp}+\mathrm{Sc}+\mathrm{LAB} 2$. Significant differences for petunidin 3-O-(6-acetyl) glucosides were not evident among the analysed wines. Peonidin and malvidin 3-O-(6-acetyl) glucoside concentrations were highest in wines which underwent MLF. Delphinidin 3-O-(6- $p$ coumaroyl) glucoside concentrations were not significantly different among the wines. Malvidin 3-O-(6- $p$-coumaroyl) glucoside concentrations showed significant differences among wines made with a combination of $\mathrm{Sc}+\mathrm{LAB} 1, \mathrm{Hu}$ $+\mathrm{Sc}+\mathrm{LAB} 2$ and $\mathrm{Mp}+\mathrm{Sc}+\mathrm{LAB} 2$, compared to wines made with $S$. cerevisiae only. Wines that underwent MLF were highest in malvidin 3-O-(6- $p$-coumaroyl) glucoside concentrations, except wines made with a combination of $\mathrm{Mp}+\mathrm{Sc}+\mathrm{LAB} 1$.

\section{Treatment effect on sensory attributes}

Wines made with a combination of $\mathrm{Mp}+\mathrm{Sc}$ and $\mathrm{Hu}+\mathrm{Sc}$ were significantly different in acidity compared to the Sconly wines (Table 3). Acidity was significantly different among wines made with $S$. cerevisiae only and wines that underwent MLF. Wines that underwent MLF were slightly less acidic but still relatively balanced compared to the yeast-only wines.

Wines made with a combination of $\mathrm{Hu}+\mathrm{Sc}+\mathrm{LAB} 1$ and $\mathrm{Mp}+\mathrm{Sc}+\mathrm{LAB} 1$ scored significantly higher in mouthfeel compared to wines made with $S$. cerevisiae only. There were also significant differences in mouthfeel between wines made with a combination of $\mathrm{Mp}+\mathrm{Sc}$ and wines made with a combination of $\mathrm{Mp}+\mathrm{Sc}$ that underwent MLF. Wines made with a combination of $\mathrm{Mp}+\mathrm{Sc}$ and $\mathrm{Hu}+\mathrm{Sc}$ scored lower in mouthfeel compared to wines that underwent MLF. The wines did not differ significantly in terms of astringency.

The judges scored the reference wines ( $S$. cerevisiae only) higher in bitterness compared to wines made with a combination of $\mathrm{Mp}+\mathrm{Sc}$ and $\mathrm{Hu}+\mathrm{Sc}$ and wines that underwent MLF.

Wines that underwent MLF showed significantly higher overall quality scores compared to the reference wines (Sc only). Wines made with a combination of $\mathrm{Hu}+\mathrm{Sc}$ also scored significantly higher in overall quality compared to the reference wines (Sc only). 


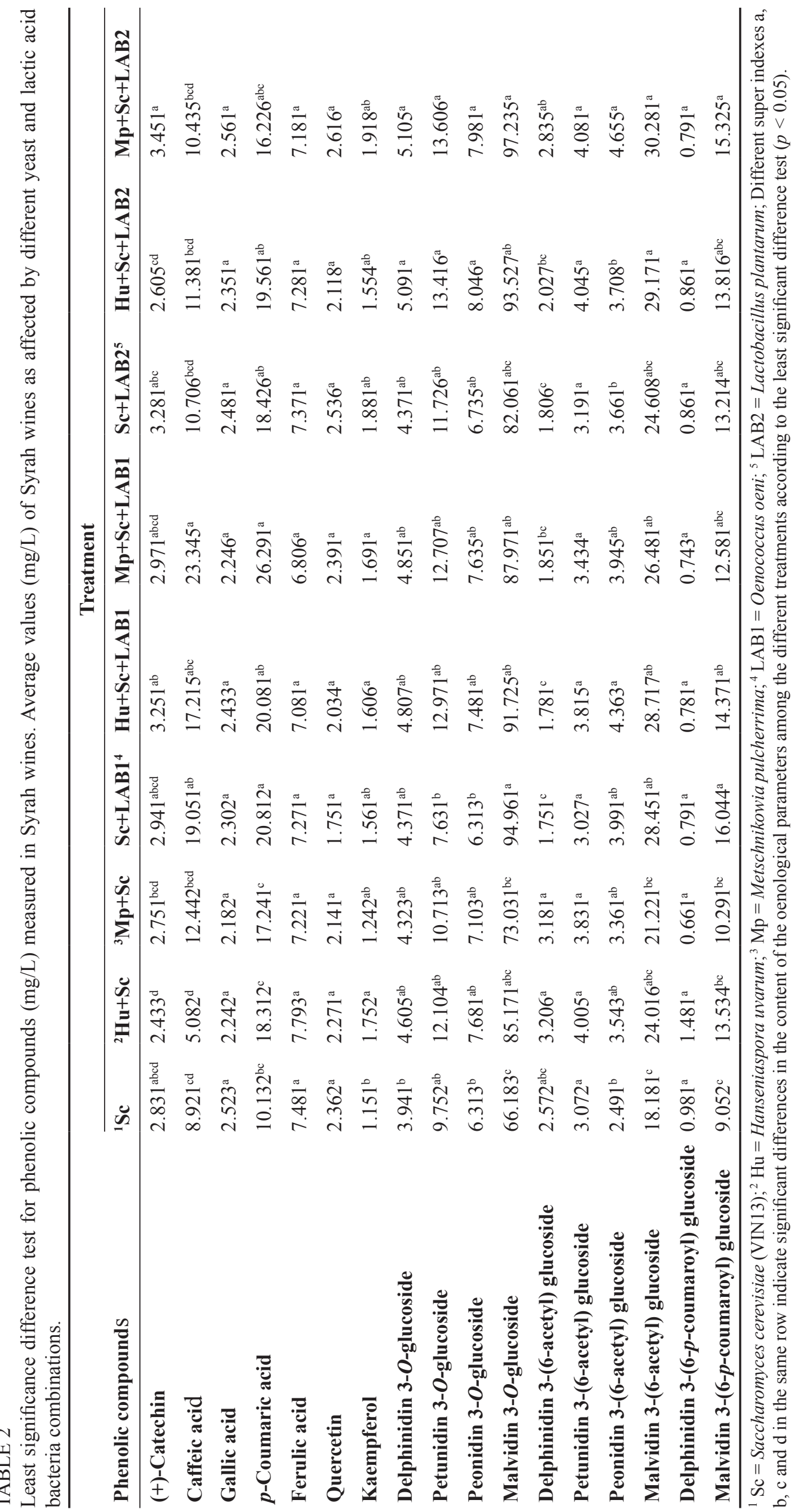




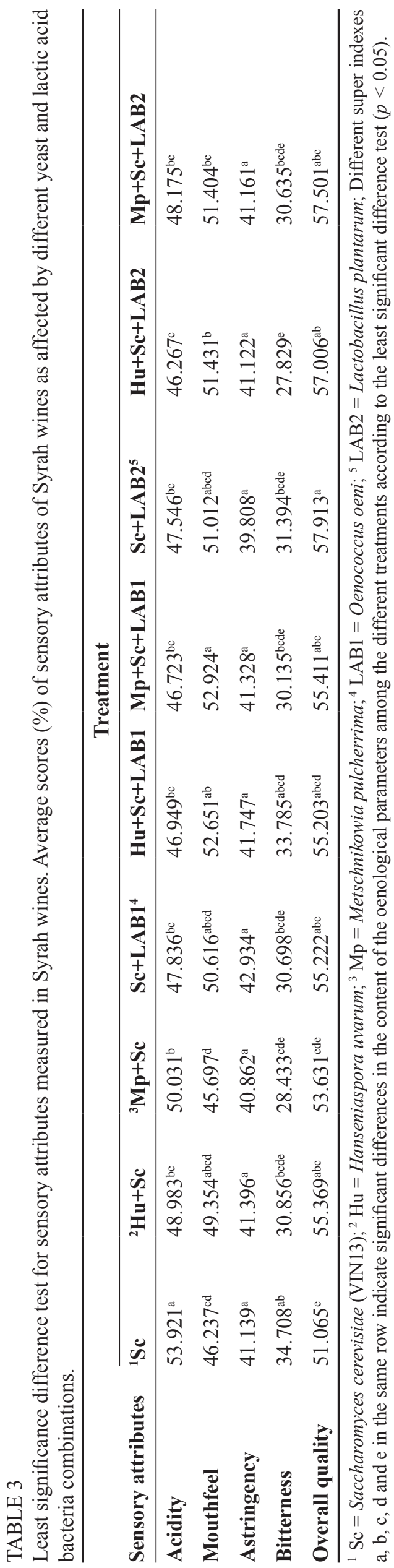

\section{DISCUSSION}

This trial, which included mixed cultures of nonSaccharomyces and S. cerevisiae and LAB species in Syrah must, showed that wines fermented with combinations of yeast and LAB (except Sc + LAB1 and Sc + LAB2) contained slightly lower ethanol concentrations than the $S$. cerevisiae reference wines (Table 1). These results agree with findings by Comitini et al. (2011), who reported higher ethanol concentrations and higher total acidity in wines (small-scale fermentation) made with $S$. cerevisiae only than in wines made with a combination of M. pulcherrima and $S$. cerevisiae. However, there were no significant differences in ethanol concentrations in wines made with $S$. cerevisiae only and a combination of Sc + LAB1. This is in agreement with work by Benito et al. (2015), who also reported no significant differences in ethanol concentrations in wines made with $S$. cerevisiae only and wines made with a combination of $S$. cerevisiae $+O$. oeni.

Lactic acid bacteria, S. cerevisiae, H. uvarum and M. pulcherrima in combination positively affected the phenolic acid concentrations of the wines (Table 2). Wines that were made with a combination of $\mathrm{Sc}+\mathrm{LAB} 1$ and $\mathrm{Mp}$ $+\mathrm{Sc}+\mathrm{LAB} 1$ were higher in caffeic and $p$-coumaric acids respectively compared to wines made with a combination of yeasts only. This is in agreement with work by Hernández et al. (2006; 2007), who found that wines that underwent MLF (LAB1 and LAB2) after initial inoculation with $S$. cerevisiae were higher in caffeic and $p$-coumaric acids, $(+)$-catechin and quercetin compared to wines made with $S$. cerevisiae only. Chescheir et al. (2015) reported increased concentrations of caffeic and $p$-coumaric acids in Pinot noir wines made with $O$. oeni strain Viniflora ${ }^{\circledR}$ oenos compared to wines made with $S$. cerevisiae only.

Variation in phenolic compound concentrations were noticeable among wines made with the non-Saccharomyces yeasts and LAB. Wines made with $S$. cerevisiae only were associated with lower total anthocyanin and phenolic acid concentrations compared to wines made with a combination of non-Saccharomyces yeast and LAB. Wines made with a combination of $\mathrm{Mp}+\mathrm{Sc}+\mathrm{LAB} 2$ were associated with the highest total anthocyanin concentrations, followed by wines made with a combination of $\mathrm{Hu}+\mathrm{Sc}+\mathrm{LAB} 2$. This is in agreement with work by Vivas et al. (1997b), who reported a positive effect of MLF (LAB2) on red wine composition and quality.

The results also show that wines made with a combination of $\mathrm{Mp}+\mathrm{Sc}+\mathrm{LAB} 2$ and $\mathrm{Hu}+\mathrm{Sc}+\mathrm{LAB} 2$ were higher in delphinidin and malvidin 3-O-glucoside concentrations compared to wines made without LAB. Burns and Osborne (2015) reported higher peonidin, petunidin, delphinidin and malvidin 3-O-glucoside concentrations in Pinot noir wines that had undergone MLF compared to wines made with S. cerevisiae only.

The combinations of yeasts and LAB affected the acid balance of the wine (Table 3). Wines that underwent MLF showed lower acidity compared to wines that did not undergo MLF. Wines that underwent MLF scored higher in mouthfeel than $S$. cerevisiae-only wines and wines made with a combination of yeasts. Wines made with $\mathrm{Mp}$ $+\mathrm{Sc}+\mathrm{LAB} 1$ had the highest caffeic and $p$-coumaric acid 
concentrations and scored highest in mouthfeel. There was no clear correlation between the concentrations of phenolic compounds and taste attributes such as the astringency and bitterness of wines. However, increased anthocyanin concentrations, i.e. colour, were obtained in wines made with non-Saccharomyces yeast and wines that underwent MLF. These wines also scored highest in overall quality. Wines that were highest in overall quality were also highest in flavonols, flavan-3-ols and anthocyanins.

\section{CONCLUSIONS}

This paper reports on the effects of non-Saccharomyces yeast and LAB on the phenolic compound concentrations as well as selected sensory attributes of Syrah wines during alcoholic fermentation. Inoculation with a combination of yeast and lactic acid bacteria proved advantageous for red wine colour compared to yeast only. Syrah wines made with $S$. cerevisiae only were highest in perceived acidity, followed by wines made with a combination of $M$. pulcherrima and $S$. cerevisiae. Wines made with a combination of $M$. pulcherrima, S. cerevisiae and $O$. oeni scored highest in mouthfeel, whereas wines made with a combination of $S$. cerevisiae and $O$. oeni scored highest in astringency. The least astringent wines made with a combination of $S$. cerevisiae and L. plantarum. These wines scored highest in overall quality. Syrah wines made with a combination of $M$. pulcherrima, S. cerevisiae and L. plantarum scored second highest in overall quality.

Syrah wines made with $M$. pulcherrima, S. cerevisiae and L. plantarum were highest in chromatic properties, i.e. total anthocyanin (colour) concentrations, followed by H. uvarum in combination with $S$. cerevisiae and L. plantarum. Syrah wines made with $S$. cerevisiae in combination with $O$. oeni, and $M$. pulcherrima in combination with $S$. cerevisiae and $O$. oeni, produced wines with the highest phenolic acid concentrations.

Improved colour of Syrah wine may be achievable by sequential inoculation with $S$. cerevisiae + L. plantarum and M. pulcherrima, and in combination with S. cerevisiae + L. plantarum and H. uvarum. Improved colour suggests low adsorption of anthocyanins on yeast and bacterial cell walls, which has a delayed effect on the polymerisation process. Increased anthocyanin concentrations in the Syrah wine indicate that adsorption by yeast and bacterial cell walls was minimal and therefore had a positive effect on wine colour and overall quality. Low adsorption of anthocyanins on yeast cell walls could be useful to produce wines with improved colour and quality where winemakers use grape cultivars with low anthocyanin levels in the grape skin.

\section{LITERATURE CITED}

Alberto, M.R., Arena, M.E. \& Manca de Nadra, M.C., 2007. Putrescine production from agmatine by Lactobacillus hilgardii: Effect of phenolic compounds. Food Cont. 18, 898-903.

Bautista-Ortín, A.B., Romero-Cascales, I., Fernández-Fernández, J.I., López-Roca, J.M. \& Gómez-Plaza, E., 2007. Influence of the yeast strain on Monastrell wine colour. Inn. Food Sci. Emerg. Technol. 8, 322-328.
Benito, Á., Calderón-Fernandez, F., Palomero, F. \& Benito, S., 2015. Combined use of selected Schizosaccharomyces pombe and Lachancea thermotolerans yeast as an alternative to the traditional malolactic fermentation in red wine production. Molecules 20, 9510-9523.

Burns, T.R. \& Osborne, J.P., 2015. Loss of Pinot noir wine colour and polymeric pigment after malolactic fermentation and potential causes. Am. J. Enol. Vitic. 66, 130-137.

Caridi, A., Cufari, A., Locino, R., Palumbo, R. \& Tedesco, I., 2004. Influence of yeast on polyphenol composition of wines. Food Technol. Biotechnol. 42, 37-40.

Chescheir, S., Philbin, D. \& Osborne, J.P., 2015. Impact of Oenococcus oeni on wine hydroxycinnamic acids and volatile phenol production by Brettanomyces bruxellensis. Am. J. Enol. Vitic. 66, 357-362.

Cheynier, V., Arellano, I.H., Souquet, J.M. \& Moutounet, M., 1997. Estimation of the oxidative changes in phenolic compounds of Carignane during winemaking. Am. J. Enol. Vitic. 48, 225-228.

Comitini, F., Gobbi, M., Domizio, P., Romani, C., Lencioni, L., Mannazzu, I. \& Ciani, M., 2011. Selected non-Saccharomyces wine yeasts in controlled multistarter fermentations with Saccharomyces cerevisiae. Food Microbiol. $28,873-882$.

Di Stefano, R., Borsa, D. \& Gentilini, N., 1994. Extraction of anthocyanins from the grape berry during fermentation. L'Enotecnico 30, 75-83.

Du Plessis, H.W., Du Toit, M., Hoff, J.W., Hart, R.S., Ndimba, B.K. \& Jolly, N.P., 2017. Characterisation of non-Saccharomyces yeasts using different methodologies and evaluation of their compatibility with malolactic fermentation. S. Afr. J. Enol. Vitic. 38, 46-63.

Du Toit, M., Engelbrecht, L., Lerm, E. \& Krieger-Weber, S., 2010. Lactobacillus: The next generation of malolactic fermentation starter cultures - an overview. Food Bioprocess Technol. 4, 876-906.

Eglinton, J., Griesser, M., Henschke, P., Kwiatkowski, M., Parker, M. \& Herderich, M., 2004. Yeast-mediated formation of pigmented polymers in red wine. In: Waterhouse, A.L. \& Kennedy, J.A. (eds). Red wine colour: Exploring the mysteries. American Chemical Society, Washington, DC. pp. $7-21$.

Fulcrand, H., Dueñas, M., Salas, E. \& Cheynier, V., 2006. Phenolic reactions during winemaking and aging. Am. J. Enol. Vitic. 57, 289-297.

García-Ruiz, A., Bartolomé, B., Martínez-Rodríguez, A., Pueyo, E., Martín Álvarez, P.J. \& Moreno-Arribas, M.V., 2008. Potential of phenolic compounds for controlling lactic acid bacteria growth in wine. Food Control $19,835-841$.

Gawel, R. \& Godden, P.W., 2008. Evaluation of the consistency of wine quality assessments from expert wine tasters. Aust. J. Grape Wine Res. 14, $1-8$.

Hernández, L.F., Espinosa, J.C. \& Fernández-González, A.B., 2003. ß-glucosidae activity in a Saccharomyces cerevisiae wine strain. Int. J. Food Microbiol. 80, 171-176.

Hernández, T., Estrella, I., Carlavilla, D., Martín-Álvarez, P.J. \& MorenoArribas, M.V., 2006. Phenolic compounds in red wine subjected to industrial malolactic fermentation and ageing on lees. Anal. Chim. Acta 563, 116-125.

Hernández, T., Estrella, I., Pérez-Gordo, M., Alegría, E.G., Tenorio, C., Ruiz-Larrea, F. \& Moreno-Arribas, M.V., 2007. Contribution of malolactic fermentation by Oenococcus oeni and Lactobacillus plantarum to the changes in the nonanthocyanin polyphenolic composition of red wine. J. Agric. Food Chem. 55, 5260-5266.

Jolly, N.P., Varela, C. \& Pretorius, I.S., 2014. Not your ordinary yeast: NonSaccharomyces yeasts in wine production uncovered. FEMS Yeast Res. 14, 215-237. 
Knoll, C., Divol, B. \& Du Toit, M., 2008. Influence of phenolic compounds on the activity of nisin and pediocin PA-1. Am. J. Enol. Vitic. 59, 418-421.

Lerm, E., Engelbrecht, L. \& Du Toit, M., 2010. Malolactic fermentation: The ABC's of MLF. S. Afr. J. Enol. Vitic. 31, 186-212.

Lorrain, B., Ky, I., Pechamat, L. \& Teissedre, P-L., 2013. Evolution of analysis of polyphenols from grapes, wines and extracts. Molecules 18, 1076-1100.

Manzanares, P., Rojas, V., Genoves, S. \& Valles, S., 2000. A preliminary search for anthocyanin- $\beta$-D-glucosidase activity in non-Saccharomyces wine yeasts. Int. J. Food Sci. Tech. 35, 95-103.

Mateus, N., Machado, J.M. \& De Freitas, V., 2002. Development changes of anthocyanins in Vitis vinifera grapes grown in the Douro Valley and concentration in respective wines. J. Sci. Food Agric. 82, 1689-1695.

Mazza, E. \& Francis, F.J., 1995. Anthocyanins in grapes and grape products. Crit. Rev. Food Sci. Nutr. 35, 341-371.

Medina, K., Boido, E., Dellacassa, E. \& Carrau, F., 2005. Yeast interaction with anthocyanins during red wine fermentation. Am. J. Enol. Vitic. 56, 104-109.

Minnaar, P.P., Ntushelo, N.S., Ngqumba, Z., Van Breda, V. \& Jolly, N.P., 2015. Effect of Torulaspora delbrueckii yeast on the anthocyanin and flavanol concentrations of Cabernet franc and Pinotage wines. S. Afr. J. Enol. Vitic. 36, 50-58.

Morata, A., Benito, S., Loira, I., Palomera, F., González, M.C. \& SuárezLepe, J.A., 2012. Formation of pyranoanthocyanins by Schizosaccharomyces pombe during the fermentation of red must. Int. J. Food Microbiol. 159, 47-53.

Morata, A., Gómez-Cordovés, M.C., Suberviola, J., Bartolomé, B., Colomo, B. \& Suárez, J.A., 2003. Adsorptions of anthocyanins on yeast cell walls during the fermentation of red wines. J. Agric. Food Chem. 51, 4048-4088.

Nguela, J.M., Sieczkowski, N., Roi, S. \& Vernhet, A., 2015. Sorption of grape proanthocyanidins and wine polyphenols by yeasts, inactivated yeasts and yeast cell walls. J. Agric. Food Chem. 63, 660-670.

Ott, R.L., 1998. An introduction to statistical methods and data analysis. Belmont, CA, Duxbury Press. Pp. $807-837$.

Padilla, B., Gil., J.V. \& Manzamares, P., 2016. Past and future of nonSaccharomyces yeasts: From spoilage microorganisms to biotechnological tools for improving wine aroma complexity. Front. Microbiol. 7, 1-20.
Reguant, C., Bordons, A., Arola, L. \&. Rozès, N., 2000. Influence of phenolic compounds on the physiology of Oenococcus oeni from wine. J. Appl. Microbiol. 88, 1065-1071.

Ribéreau-Gayon, P., Glories, Y., Maujean, A. \& Du Bourdieu, D., 2006 ( $\left.2^{\text {nd }} \mathrm{ed}\right)$. Handbook of enology: The chemistry of wine stabilisation and treatment. John Wiley \& Sons Inc, New York.

Rozès, N., Arola, L. \& Bordons, A., 2003. Effect of phenolic compounds on the co-metabolism of citric acid and sugars by Oenococcus oeni from wine. Lett. Appl. Microbiol. 36, 337-341.

Salmon, J.-M., 2006. Interactions between yeast, oxygen and polyphenols during alcoholic fermentations: Practical implications. LWT Food Sci. Technol. 39, 959-965.

SAS Institute Inc., n.d. SAS/STAT software. SAS Institute Inc., Cary, NC, USA.

Schwarz, M., Wabnitz, T. \& Winterhalter, P., 2003. Pathway leading to the formation of anthocyanin-vinylphenol adducts and related pigments in red wines. J. Agric. Food Chem. 51, 3682-3687.

Shapiro, S.S. \& Wilk, M.B., 1965. An analysis of variance test for normality (complete samples). Biometrika 52, 591-611.

Song, J., Smart, R., Wang, H., Dambergs, B., Sparrow, A. \& Qian, M.C., 2015. Effects of grape bunch sunlight exposure and UV radiation on phenolics and volatile composition of Vitis vinifera $\mathrm{L}$. cv. Pinot noir. Food Chem. 173, 424-731.

Vivas, N., Lonvaud-Funel, A. \& Glories, Y., 1997a. Effect of phenolic acids and anthocyanins on growth, viability and malolactic activity of a lactic acid bacterium. Food Microbiol. 14, 291-300.

Vivas, N., Lonvaud-Funel, A., Glories, Y. \& Augustin, M., 1997b. Effect of malolactic fermentation in barrels and tanks on red wine composition and quality. Practical Winery and Vineyard May/June, 68-77.

Waterhouse, A.L., Price, S.F. \& McCord, J.D., 1999. Reversed phase high-performance liquid chromatography methods for analysis of wine polyphenols. Meth. Enzym. 299, 113-122.

South African Wine Industry Information and Systems (SAWIS), 2015. Final report - Macro-economic impact of the wine industry on the South African economy (also with reference to the impacts on the Western Cape). Pretoria: Conningarth Economists.

WOSA (Wines of South Africa), n.d. Statistics [Online]: http://www.wosa. co.za/The-Industry/Statistics/World-Statistics [accessed on 07 Feb 2017]. 